\title{
Predicting the UHE photon flux from GZK-interactions of hadronic cosmic rays using CRPropa 3
}

\author{
Anna Bobrikova, ${ }^{a, b}$ Marcus Niechciol, ${ }^{a}$ Markus Risse ${ }^{a}$ and Philip Ruehl ${ }^{a, *}$ \\ ${ }^{a}$ University of Siegen \\ Walter-Flex-Str. 3, Siegen, Germany \\ ${ }^{b}$ now at University of Turku \\ 20014 Turun yliopisto, Finland \\ *E-mail: ruehl@hep.physik.uni-siegen.de
}

The spectrum of ultra high energy (UHE) cosmic rays as measured by the Pierre Auger Observatory indicates a strong flux suppression above $50 \mathrm{EeV}$. The origin of this suppression is still unclear. One possible explanation is the Greisen-Zatsepin-Kuzmin (GZK) process, in which UHE cosmic rays interact with the cosmic microwave background. Indirect evidence for the GZK-process could be provided by the search for UHE photons produced in such an interaction. A signal of UHE photons could not yet be identified among the cosmic rays. Hence, upper limits on the UHE photon flux have been derived from experimental data of various experiments. In order to interpret these limits, theoretical predictions are needed. In this contribution, new predictions on the UHE photon flux above $10^{15.8} \mathrm{eV}$ are derived assuming different compositions of the initial cosmic rays. The simulation study has been done using CRPropa 3. Latest results regarding the extragalactic medium and the mass composition of cosmic rays as measured by the Pierre Auger Observatory are taken into account. For all compositions, the predictions stay below the current upper limits on the UHE photon flux derived from experimental data. The main uncertainties on the predictions originate from the lack of knowledge about the initial source spectra of UHE cosmic rays.

$37^{\text {th }}$ International Cosmic Ray Conference (ICRC 2021)

July 12 th - 23rd, 2021

Online - Berlin, Germany

\footnotetext{
${ }^{*}$ Presenter
} 


\section{Introduction}

Measurements of the UHE cosmic ray spectrum at the ground level taken by the Pierre Auger Observatory [1] indicate a suppression in the spectrum at about $50 \mathrm{EeV}$ [2]. The origin of this suppression is still unclear. One possible way to explain this observation is to assume a limitation of the sources which might not be able to provide enough energy to accelerate particles well beyond $50 \mathrm{EeV}$. Another explanation could be provided by interactions of the CRs during their propagation from their source to earth. In particular the so-called Greisen-Zatsepin-Kuzmin (GZK) process $[3,4]$

$$
p+\gamma_{\mathrm{CMB}} \rightarrow \Delta^{+}(1232) \rightarrow\left\{\begin{array}{l}
p+\pi^{0} \\
n+\pi^{+}
\end{array},\right.
$$

in which ultra high energy proton loose energy in interactions with the photons of the cosmic microwave background is also able to explain the observed spectral behavior. Indirect evidence for the GZK-process could be provided by the search for ultra high energy (UHE) photons that are produced in such an interaction through the decay of the neutral pions in the final state. A signal of UHE photons could not yet be identified among the cosmic ray flux. Upper limits on the diffuse flux of UHE photons have been derived by various experiments [5-8].

In order to interpret these upper limits, theoretical predictions are needed. Predictions of the photon flux above $1 \mathrm{EeV}$ as expected from the propagation of UHECR from their sources towards earth as caused by the GZK-process have been derived in previous studies $[9,10]$. While using detailed models for the particle propagation through the extragalactic medium, comparably simple benchmark scenarios regarding the mass composition of CRs have been applied therein due to a lack of experimental evidence. Recent results by the Pierre Auger Observatory, however, limit the credible phase space of cosmic rays composition and spectrum at the sources. Here, this new evidence together with recent models of the extragalactic medium and particle interactions are used to revise the predictions about the UHE photon flux and extend their valid energy range down to $10^{15.8} \mathrm{eV}$.

\section{Simulation Setup with CRPropa 3}

To simulate the generation of UHE photons through GZK interactions of hadronic cosmic rays, the CRPropa 3 simulation framework has been used. CRPropa 3 is a numerical tool developed to calculate different astrophysical predictions of cosmic ray propagation [11]. It includes combined knowledge of multiple aspects of cosmic ray sources and interactions, magnetic fields, photon backgrounds, and cosmological effects. Its design is modular and adapted for multi-core calculations by propagating each particle independently.

Various boundary conditions of the propagation setup can be fine-tuned through the python interface of CRPropa 3. For this study of GZK photons a relatively simple setup involving a onedimensional propagation scenario has been chosen with a uniform magnetic field perpendicular to the direction of motion. In compliance with the more detailed models of extragalactic magnetic fields proposed in [12] and [13], an order-of-magitude estimate of $B=1 \mathrm{nG}$ has been chosen 
for the absolute value of the magnetic field. Since the propagation is implemented as a onedimensional process, synchrotron radiation in the magnetic field only serves as an energy-loss process and deflections are not taken into account. The sources of cosmic rays are also placed in a one-dimensional distribution between $4 \mathrm{Mpc}$ and $2800 \mathrm{Mpc}$ (comoving distance) from the observer following the density evolution $\rho(z) \propto(1+z)^{m}$ with redshift $z$ and $m=3.4$ corresponding to observations of the star formation rate for $z<1[14,15]$. Due to the lack of a sufficiently strong multipole structure of UHE cosmic rays, sources closer than $4 \mathrm{Mpc}$ are not considered. Sources beyond $2800 \mathrm{Mpc}$, on the other hand, are not expected to contribute significantly to the UHE photon flux on earth. The sources are configured to emit cosmic rays directly in the direction of the observer. For geometrical reasons, this setup is equivalent to an isotropic emission in three dimensions for isotropic source distributions. The background photon fields are the key ingredient for studying the GZK effect. In addition to the cosmic microwave background (CMB) also the universal radio background (URB) and the infrared background light (IRB) have been taken into account. For the latter, the models from Gilmore et al. [16] (IRB) and Protheroe and Biermann [17] (URB) were used.

The particle interactions that are taken into account in the propagation step of CR nuclei are photopion production and photodisintegration of hadronic primaries in interactions with photons from the CMB and IRB and, in the case of hadrons with limited lifetime, also nuclear decay. For CR photons, pair production, double pairproduction and triple pairproduction as well as inverse comptonscattering of electrons with the background radiation fields has been included in the simulation. For all interaction processes, particles down to a minimum energy of $10^{15.8} \mathrm{eV}$ were kept and further propagated except for neutrinos which typically completely decouple from the electromagnetic cascade and are of no further interest for this study.

\section{Composition and Spectrum of Cosmic Rays at the Source}

The analysis of $X_{\max }$, which is the depth of maximum development of the shower in the atmosphere, at the Pierre Auger Observatory shows that the composition of primary cosmic rays at earth changes for different energies [18]. For instance, the CR flux on Earth at energy less than $1 \mathrm{EeV}$ appears to be produced from light primary CRs, and for the highest energies heavy nuclei can describe the observations. However, the CR flux on Earth in the energy range approximately from $1 \mathrm{EeV}$ to $100 \mathrm{EeV}$ is estimated to be a result of the propagation of mixed cosmic rays from their sources towards Earth [19]. In this energy range, a combined fit of a set of source parameters to Auger data has been performed [19]. The source parameters that have been fitted describe the spectral shape and the elemental fractions of initial cosmic rays. Instead of individual elements, only five elemental classes are assumed characterized by their mass. The classes are substituted by representative elements ${ }^{1} \mathrm{H},{ }^{4} \mathrm{He},{ }^{14} \mathrm{~N},{ }^{28} \mathrm{Si}$ and ${ }^{56} \mathrm{Fe}$ to simplify the parameter space. The spectral shape of the initial cosmic rays is modeled as a powerlaw with spectral index $\alpha$ and an exponential suppression with rigidity-dependent cut-off $R_{\text {cut-off: }}$

$$
\frac{\mathrm{d} N_{A}}{\mathrm{~d} E} \propto f_{A} E^{-\alpha} f_{\text {cut-off }}\left(E, Z_{A} R_{\text {cut-off }}\right)
$$




$$
f_{\text {cut-off }}\left(E, Z_{A} R_{\text {cut-off }}\right)= \begin{cases}1 & \left(E<Z_{A} R_{\text {cut-off }}\right) \\ \exp \left(1-\frac{E}{Z_{A} R_{\text {cut-off }}}\right) & \left(E>Z_{A} R_{\text {cut-off }}\right) .\end{cases}
$$

Here, $f_{A}$ is a free parameter which is the compositional fraction of the elemental class represented by the mass number $A$ and the corresponing charge number $Z_{A}$. This setup of boundary conditions leaves 7 free parameters for the fitting procedure. As the result of two different fitting procedures, i.e. the likelihood scanning method and posterior sampling, three parameter sets were extracted as possible source scenarios. Those parameter sets, which are the main and second minimum of the likelihood scanning method (scenario 1 and 2, respectively) and the best fit scenario of the posterior sampling method (scenario 3), are adopted here in order to derive the associated GZK-photon flux. The resulting parameter sets of the fitting procedure are compiled in Table 1.

\begin{tabular}{||l|ccccccc||}
\hline Parameter & $\alpha$ & $\log _{10}\left(R_{\text {cut-off }} / e V\right)$ & $f_{\mathrm{H}}(\%)$ & $f_{\mathrm{He}}(\%)$ & $f_{\mathrm{N}}(\%)$ & $f_{\mathrm{Si}}(\%)$ & $f_{\mathrm{Fe}}(\%)$ \\
\hline Scenario 1 & 0.96 & 18.68 & 0.0 & 67.3 & 28.1 & 4.6 & 0.0 \\
Scenario 2 & 2.04 & 19.88 & 0.0 & 0.0 & 79.8 & 20.2 & 0.0 \\
Scenario 3 & 1.22 & 18.72 & 6.4 & 46.7 & 37.5 & 9.4 & 0.0 \\
\hline
\end{tabular}

Table 1: The fit results of the seven source parameters from the combined fit analysis [19].

\section{GZK Photon Predictions}

For the three CR compositions scenarios that are favored by the data of the Pierre Auger Observatory, dedicated simulations have been performed with CRPropa 3 using the simulation setup described in Sec. 2. Secondary photons that are produced in GZK-interactions are simulated down to energies of $10^{15.8} \mathrm{eV}$. The resulting integral photon spectra for each of the mixed composition scenarios are shown in Fig. 1. Here, the individual contributions of each initial nucleus type to the total spectra are displayed by a decomposition of the total spectrum. For all three scenarios, as expected from the initial compositional fractions $f_{A}$, heavier nuclei provide a significant or even dominant contribution to the resulting UHE photon flux. For composition scenarios 1 and 3, which both follow a comparably hard spectral distribution, it is interesting to note that the relative photon flux (when normalized to the initial compositional fraction) is larger for the more heavier nuclei. In other words, the UHE photon yield appears to increase for heavier inital primary mass. Hence, in contrast to previous studies of GZK photons, the most optimistic UHE photon predictions are not necessarily obtained from the lightest possible composition when the source parameters are constrained by data-driven boundary conditions with a chosen functional form given by Eqs. 2 and 3.

A comparison of the total photon flux predictions of all analysed scenarios is shown in Fig. 2. Scenario 1 leads to the most optimistic photon flux predictions while composition scenario 2 has the lowest photon yield. These overall differences can mainly be attributed to the different spectral indices. The higher rigidity cut-off of scenario 2 as compared to scenarios 1 and 3 only plays a minor role since it cannot compensate the steep powerlaw distribution. 


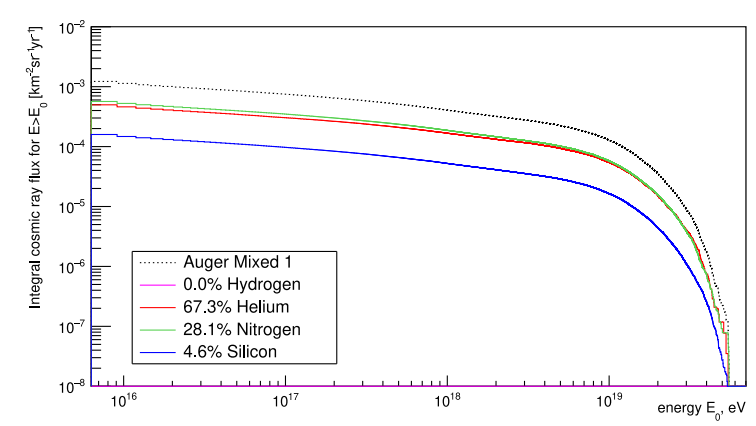

(a)

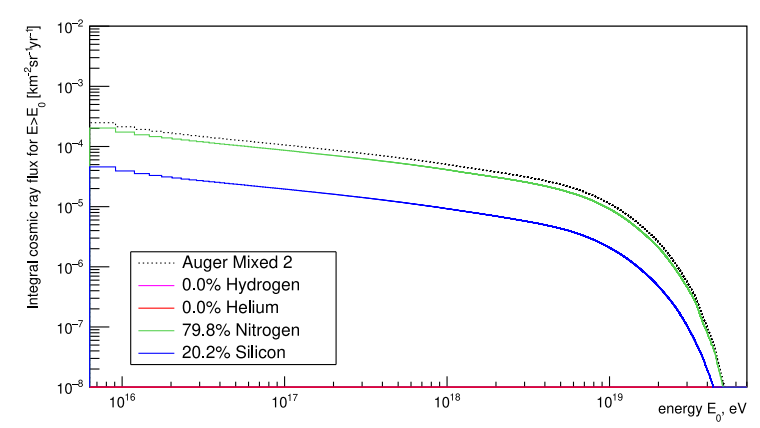

(b)

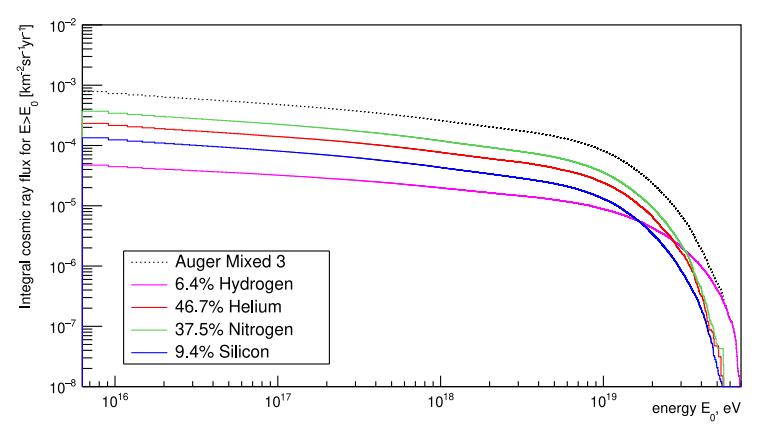

(c)

Figure 1: The contributions of individual elements to the predicted GZK photon spectra from the mixed composition scenarios that are summarized in Tab.1: (a) Scenario 1, (b) Scenario 2 and (c) Scenario 3. In each composition scenario, the contribution from initial nitrogen nuclei dominates the overall GZK photon flux.

The results are also compared in Fig. 2 to those of a previous study of pure protons [10]. The new predictions of GZK photons extend the previous ones by two orders of magnitude towards lower energies. While the source models between the previous study and this work differ in multiple parameters, the discrepancy between the model predictions is mainly driven by a high rigidity cutoff which had been assumed to be $R_{\max }=10^{21} \mathrm{eV}$ in [10]. In addition to the different source models, also upgrades to the simulation of photopion-production within CRPropa 3 itself lead to changes of the photon flux compared to previous code versions.

\section{Summary}

Using the latest results about the CR spectrum and mass composition from the Pierre Auger Observatory, new predictions of the integral flux of GZK photons above $10^{15.8} \mathrm{eV}$ have been derived using the functionality of the CRPropa 3 simulation code. Restricting the CR spectrum and composition scenario at the sources to parameter sets favored by Auger data introduces a non-trivial relation between the GZK photon flux and the chemical composition of CRs. With these data-driven boundary conditions, the lightest CR components do no longer necessarily contribute the largest GZK photon flux, indicating a more complex dependence of the GZK photon yield from the initial source parameters. 


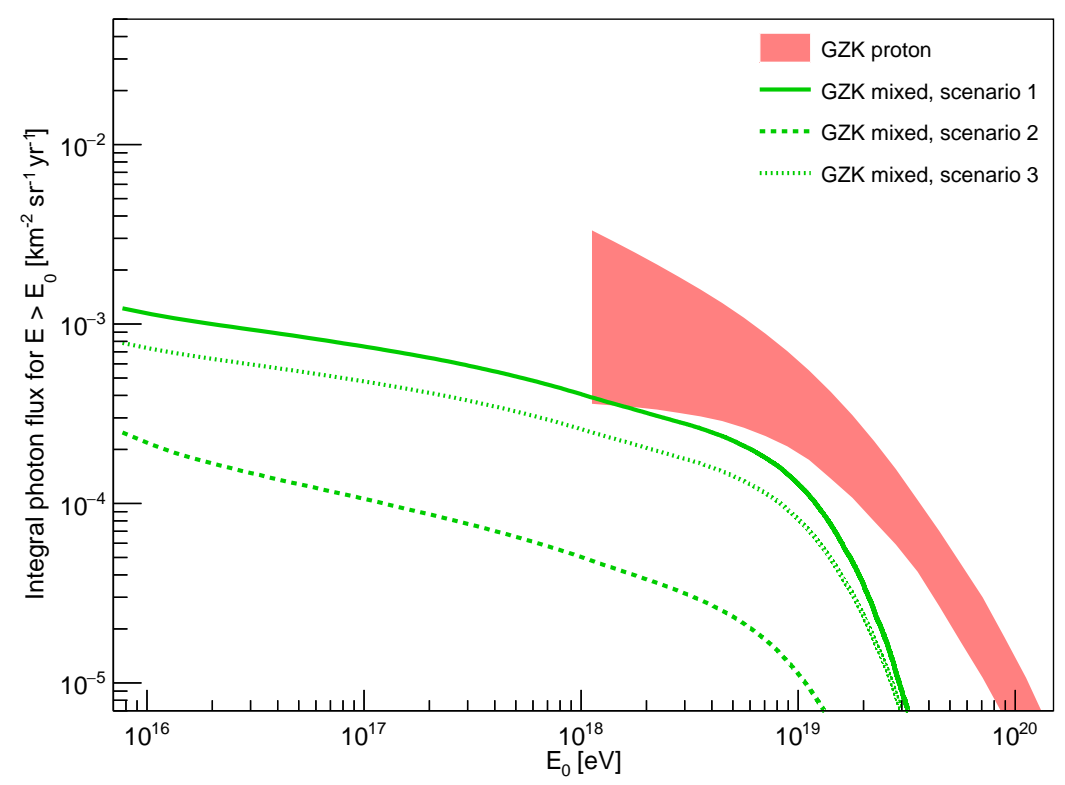

Figure 2: The GZK photon spectra that are predicted as a result of the propagation of initial cosmic rays from their sources towards earth. The green lines show the photon fluxes that are caused by the three source scenarios described in Tab. 1. The results of a preceeding study [10] of GZK photons from pure proton CRs are depicted by the shaded region.

\section{References}

[1] A. Aab [Pierre Auger Coll.], Nucl. Instrum. Meth. A 798 (2015) 172 [1502. 01323].

[2] A. Aab [Pierre Auger Coll.], Phys. Rev. Lett 125 (2020) 121106 [2008. 06488].

[3] K. Greisen, Phys. Rev. Lett. 16 (1966) 748.

[4] G. T. Zatsepin, V. A. Kuz'min, J. Exp. Teor. Phys. Lett. 4 (1966) 78-80.

[5] W. D. Apel et al., Astrophys. J. 858 (2017) 1 [1710.02889].

[6] R. Abbasi et al., Astropart. Phys. 110 (2019) 8-14 [1811.03920].

[7] Y. A. Fomin et al., Phys. Rev. D 95 (2017) 123011 [1702.08024].

[8] J. Rautenberg [Pierre Auger Coll.], Proc. 36th Int. Cosmic Ray Conf., Madison, USA (2019) PoS(ICRC2019)398 [1909.09073].

[9] G. Gelmini et al., J. Exp. Theor. Phys. 106 (2008) 1061 [astro-ph/0506128].

[10] B. Sarkar et al., Proc. 32nd Int. Cosmic Ray Conf., Beijing, China (2011) 2 (2011) 198.

[11] R. A. Batista et al., J. Cosmol. Astropart. P. 05 (2016) 38 [1603.07142].

[12] K. Dolag et al., J. Cosmol. Astropart. P. 01 (2005) 009 [astro-ph/0410419].

[13] G. Sigl et al., Phys. Rev. D 70 (2004) 043007 [astro-ph/0401084].

[14] A. M. Hopkins, J. F. Beacom, Astrophys. J. 651 (2006) 142-154 [astro-ph/0601463].

[15] H. Yüksel et al., Astrophys. J. 683 (2008) L5-L8 [0804 . 4008].

[16] R. C. Gilmore et al., Mon. Not. R. Astron. Soc. 422 (2012) 3189-3207 [1104. 0671].

[17] R. Protheroe, P. Biermann, Astropart. Phys. 6 (1996) 45-54 [astro-ph/9605119].

[18] A. Yushkov [Pierre Auger Coll.], Proc. 36th Int. Cosmic Ray Conf., Madison, USA (2019) PoS(ICRC2019)482 [1909.09073].

[19] A. Aab [Pierre Auger Coll.], J. Cosmol. Astropart. P. 04 (2017) 038 [1612.07155]. 